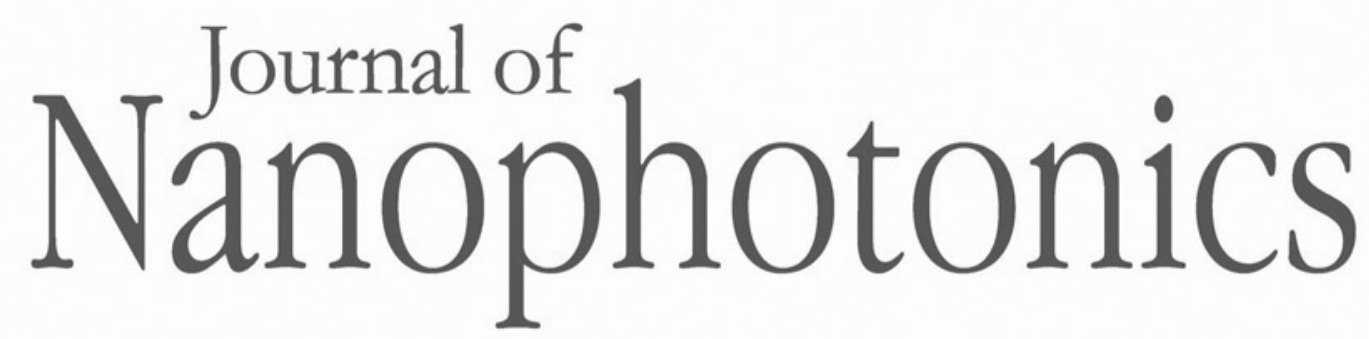

Nanophotonics.SPIEDigitalLibrary.org

\title{
Classical electromagnetic model of surface states in topological insulators
}

Akhlesh Lakhtakia

Tom G. Mackay 


\title{
Classical electromagnetic model of surface states in topological insulators
}

\author{
Akhlesh Lakhtakia ${ }^{a, *}$ and Tom G. Mackay ${ }^{a, b}$ \\ ${ }^{a}$ Pennsylvania State University, Department of Engineering Science and Mechanics, \\ NanoMM-Nanoengineered Metamaterials Group, 212 EES Building, \\ University Park, Pennsylvania 16802, United States \\ ${ }^{b}$ University of Edinburgh, School of Mathematics, Edinburgh EH9 3FD, Scotland, \\ United Kingdom
}

\begin{abstract}
A topological insulator is classically modeled as an isotropic material with a magnetoelectric pseudoscalar $\Psi$ existing in its bulk while its surface is charge free and current free. An alternative model is obtained by setting $\Psi \equiv 0$ and incorporating surface charge and current densities characterized by an admittance $\gamma$. Analysis of planewave reflection and refraction due to a topological-insulator half space reveals that the parameters $\Psi$ and $\gamma$ arise identically in the reflection and transmission coefficients, implying that the two classical models cannot be distinguished on the basis of any scattering scenario. However, as $\Psi$ disappears from the Maxwell equations applicable to any region occupied by the topological insulator, and because surface states exist on topological insulators as protected conducting states, the alternative model must be chosen. () The Authors. Published by SPIE under a Creative Commons Attribution 3.0 Unported License. Distribution or reproduction of this work in whole or in part requires full attribution of the original publication, including its DOI. [DOI: 10.1117/1.JNP.10.033004]
\end{abstract}

Keywords: admittance; magnetoelectricity; nonreciprocity; surface state; topological insulator.

Paper 15169SSP received Nov. 13, 2015; accepted for publication Jan. 13, 2016; published online Feb. 8, 2016.

\section{Introduction}

The discovery of topological insulators ${ }^{1}$ has prompted researchers in classical optics ${ }^{2-4}$ to examine electromagnetic scattering due to bound objects made of these materials, exemplified by chalcogenides such as $\mathrm{Bi}_{2} \mathrm{Se}_{3}, \mathrm{Bi}_{2} \mathrm{Te}_{3}$, and $\mathrm{Sb}_{2} \mathrm{Te}_{3}$. As a topological insulator is considered to be an isotropic material, its frequency-domain constitutive relations are formulated to contain a magnetoelectric pseudoscalar (denoted by $\Psi$ here) in addition to the permittivity scalar $\varepsilon$ and the permeability scalar $\mu$. The surface of the topological insulator is assumed to be charge free and current free, and the scattering problem can then be solved by following textbook techniques. ${ }^{5}$

Yet, according to condensed-matter theory, surface states exist on topological insulators as protected conducting states, ${ }^{2}$ and the characteristic electromagnetic responses of these materials are due to those surface states. Should then a topological insulator's optical response be modeled as due solely to either

I. the bulk constitutive parameter $\Psi$ with the surface of the topological insulator being charge free and current free, or

II. a surface parameter (denoted by $\gamma$ here) that quantifies the charge density and current density on the surface of the topological insulator?

The topological insulator possesses the permittivity $\varepsilon$ and permeability $\mu$ in both models. Both $\Psi$ and $\gamma$ are admittances, and whereas the magnetoelectric constitutive parameter $\Psi$ mediates between $\mathbf{D}$ and $\mathbf{B}$ as well as between $\mathbf{H}$ and $\mathbf{E}$ throughout the topological insulator, $\gamma$ is meaningful only on the surface of that material.

*Address all correspondence to: Akhlesh Lakhtakia, E-mail: akhlesh@psu.edu 
This communication is devoted to a comparison of models I and II, through the fundamental boundary-value problem of reflection and refraction of a plane wave. This problem is described and solved in Sec. 2 for both models. Section 3 contains a comparative discussion of the two models. Vectors are underlined. An $\exp (-i \omega t)$ dependence on time $t$ is implicit, with $\omega$ as the angular frequency and $i=\sqrt{-1}$.

\section{Fundamental Boundary-Value Problem}

Suppose that all space is divided into two mutually disjoint half spaces $\mathcal{V}_{\text {out }}=\{(x, y, z): z<0\}$ and $\mathcal{V}_{\text {in }}=\{(x, y, z): z>0\}$ separated by the surface $\mathcal{S}=\{(x, y, z): z=0\}$. We need to solve the frequency-domain macroscopic Maxwell equations:

$$
\left.\begin{array}{l}
\nabla \cdot \mathbf{B}(\mathbf{r}, \omega)=0 \\
\nabla \times \mathbf{E}(\mathbf{r}, \omega)-i \omega \mathbf{B}(\mathbf{r}, \omega)=\mathbf{0} \\
\nabla \cdot \mathbf{D}(\mathbf{r}, \omega)=0 \\
\nabla \times \mathbf{H}(\mathbf{r}, \omega)+i \omega \mathbf{D}(\mathbf{r}, \omega)=\mathbf{0}
\end{array}\right\}
$$

in $\mathcal{V}_{\text {out }}$ and $\mathcal{V}_{\text {in }}$ separately, and impose boundary conditions on $\mathcal{S}$. It is possible to do so for models I and II together.

Let the half space $\mathcal{V}_{\text {out }}$ be vacuous so that the constitutive equations

$$
\mathbf{D}(\mathbf{r}, \omega)=\varepsilon_{0} \mathbf{E}(\mathbf{r}, \omega), \quad \mathbf{H}(\mathbf{r}, \omega)=\mu_{0}^{-1} \mathbf{B}(\mathbf{r}, \omega), \quad \mathbf{r} \in \mathcal{V}_{\text {out }},
$$

hold, $\varepsilon_{0}$ being the permittivity and $\mu_{0}$ being the permeability of free space. Equation (1) can then be written as

$$
\left.\begin{array}{l}
\nabla \cdot \mathbf{B}(\mathbf{r}, \omega)=0 \\
\nabla \times \mathbf{E}(\mathbf{r}, \omega)-i \omega \mathbf{B}(\mathbf{r}, \omega)=\mathbf{0} \\
\nabla \cdot \mathbf{E}(\mathbf{r}, \omega)=0 \\
\nabla \times \mathbf{B}(\mathbf{r}, \omega)+i \omega \mu_{0} \varepsilon_{0} \mathbf{E}(\mathbf{r}, \omega)=\mathbf{0}
\end{array}\right\}, \quad \mathbf{r} \in \mathcal{V}_{\text {out }}
$$

in terms of the primitive field phasors $\mathbf{E}(\mathbf{r}, \omega)$ and $\mathbf{B}(\mathbf{r}, \omega)$.

The frequency-domain constitutive relations of the material occupying $\mathcal{V}_{\text {in }}$ are

$$
\left.\begin{array}{l}
\mathbf{D}(\mathbf{r}, \omega)=\varepsilon(\omega) \mathbf{E}(\mathbf{r}, \omega)+\Psi(\omega) \mathbf{B}(\mathbf{r}, \omega) \\
\mathbf{H}(\mathbf{r}, \omega)=\mu^{-1}(\omega) \mathbf{B}(\mathbf{r}, \omega)-\Psi(\omega) \mathbf{E}(\mathbf{r}, \omega)
\end{array}\right\}, \quad \mathbf{r} \in \mathcal{V}_{\mathrm{in}},
$$

where $\varepsilon, \mu$, and $\Psi$ are functions of $\omega$. Equation (4) allows us to accommodate model I. After substituting Eq. (4) into Eq. (1), we get

$$
\left.\begin{array}{l}
\nabla \cdot \mathbf{B}(\mathbf{r}, \omega)=0 \\
\nabla \times \mathbf{E}(\mathbf{r}, \omega)-i \omega \mathbf{B}(\mathbf{r}, \omega)=\mathbf{0} \\
\nabla \cdot \mathbf{E}(\mathbf{r}, \omega)=0 \\
\nabla \times \mathbf{B}(\mathbf{r}, \omega)+i \omega \mu(\omega) \varepsilon(\omega) \mathbf{E}(\mathbf{r}, \omega)=\mathbf{0}
\end{array}\right\}, \quad \mathbf{r} \in \mathcal{V}_{\mathrm{in}} .
$$

Let us note that $\Psi$ does not appear in the Maxwell equations applied to $\mathcal{V}_{\text {in }}$ after the convenient but inessential induction field phasors $\mathbf{D}(\mathbf{r}, \omega)$ and $\mathbf{H}(\mathbf{r}, \omega)$ have been translated into essential primitive field phasors $\mathbf{E}(\mathbf{r}, \omega)$ and $\mathbf{B}(\mathbf{r}, \omega)$.

When solving an electromagnetic boundary-value problem, it is common to use the boundary conditions:

$$
\left.\begin{array}{l}
\hat{\mathbf{n}}\left(\mathbf{r}_{\mathrm{S}}\right) \cdot\left[\mathbf{B}_{\text {out }}\left(\mathbf{r}_{\mathrm{S}}, \omega\right)-\mathbf{B}_{\text {in }}\left(\mathbf{r}_{\mathrm{S}}, \omega\right)\right]=0 \\
\hat{\mathbf{n}}\left(\mathbf{r}_{\mathrm{S}}\right) \times\left[\mathbf{E}_{\text {out }}\left(\mathbf{r}_{\mathrm{S}}, \omega\right)-\mathbf{E}_{\text {in }}\left(\mathbf{r}_{\mathrm{S}}, \omega\right)\right]=\mathbf{0} \\
\hat{\mathbf{n}}\left(\mathbf{r}_{\mathrm{S}}\right) \cdot\left[\mathbf{D}_{\text {out }}\left(\mathbf{r}_{\mathrm{S}}, \omega\right)-\mathbf{D}_{\text {in }}\left(\mathbf{r}_{\mathrm{S}}, \omega\right)\right]=\rho_{s}\left(\mathbf{r}_{\mathrm{S}}, \omega\right) \\
\hat{\mathbf{n}}\left(\mathbf{r}_{\mathrm{S}}\right) \times\left[\mathbf{H}_{\text {out }}\left(\mathbf{r}_{\mathrm{S}}, \omega\right)-\mathbf{H}_{\text {in }}\left(\mathbf{r}_{\mathrm{S}}, \omega\right)\right]=\mathbf{J}_{S}\left(\mathbf{r}_{\mathrm{S}}, \omega\right)
\end{array}\right\}, \quad \mathbf{r}_{\mathrm{S}} \in \mathcal{S},
$$


with the unit normal vector $\hat{\mathbf{n}}\left(\mathbf{r}_{\mathrm{S}}\right)$ at $\mathbf{r}_{\mathrm{S}} \in \mathcal{S}$ pointing into $\mathcal{V}_{\text {out }}$. The subscripts "in" and "out" indicate that the fields in $\mathcal{V}_{\text {in }}$ and $\mathcal{V}_{\text {out }}$, respectively, are being evaluated on $\mathcal{S}$. The quantities $\rho_{s}$ and $\mathbf{J}_{s}$ are the surface charge density and the surface current density, respectively. In order to accommodate model II, we set

$$
\left.\begin{array}{l}
\rho_{s}\left(\mathbf{r}_{\mathrm{S}}, \omega\right)=\gamma(\omega) \hat{\mathbf{n}}\left(\mathbf{r}_{\mathrm{S}}\right) \cdot \mathbf{B}_{\text {in }}\left(\mathbf{r}_{\mathrm{S}}, \omega\right) \\
\mathbf{J}_{s}\left(\mathbf{r}_{\mathrm{S}}, \omega\right)=-\gamma(\omega) \hat{\mathbf{n}}\left(\mathbf{r}_{\mathrm{S}}\right) \times \mathbf{E}_{\mathrm{in}}\left(\mathbf{r}_{\mathrm{S}}, \omega\right)
\end{array}\right\}, \quad \mathbf{r}_{\mathrm{S}} \in \mathcal{S},
$$

where $\gamma$ describes the surface states.

Let an arbitrarily polarized plane wave in $\mathcal{V}_{\text {out }}$ be incident on $\mathcal{S}$. Then the primitive field phasors in $\mathcal{V}_{\text {out }}$ can be written as

$$
\left.\begin{array}{rl}
\mathbf{E}(\mathbf{r}, \omega)= & \left\{\left[a_{s} \hat{\mathbf{u}}_{y}+a_{p}\left(-\hat{\mathbf{u}}_{x} \tau_{0}+\hat{\mathbf{u}}_{z} \kappa\right) / k_{0}\right] \exp \left(i \tau_{0} z\right)\right. \\
& \left.+\left[r_{s} \hat{\mathbf{u}}_{y}+r_{p}\left(\hat{\mathbf{u}}_{x} \tau_{0}+\hat{\mathbf{u}}_{z} \kappa\right) / k_{0}\right] \exp \left(-i \tau_{0} z\right)\right\} \exp (i \kappa x) \\
\mathbf{B}(\mathbf{r}, \omega)= & \frac{k_{0}}{\omega}\left\{\left[-a_{p} \hat{\mathbf{u}}_{y}+a_{s}\left(-\hat{\mathbf{u}}_{x} \tau_{0}+\hat{\mathbf{u}}_{z} \kappa\right) / k_{0}\right] \exp \left(i \tau_{0} z\right)\right. \\
& \left.+\left[-r_{p} \hat{\mathbf{u}}_{y}+r_{s}\left(\hat{\mathbf{u}}_{x} \tau_{0}+\hat{\mathbf{u}}_{z} \kappa\right) / k_{0}\right] \exp \left(-i \tau_{0} z\right)\right\} \exp (i \kappa x)
\end{array}\right\}, \quad \mathbf{r} \in \mathcal{V}_{\text {out }},
$$

where $k_{0}=\omega \sqrt{\mu_{0} \varepsilon_{0}}, \tau_{0}=+\sqrt{k_{0}^{2}-\kappa^{2}}$, and the dependences on $\omega$ are implicit. Representing the incident plane wave, the coefficients $a_{s}$ and $a_{p}$ are presumed to be known. Representing the plane wave reflected into $\mathcal{V}_{\text {out }}$, the coefficients $r_{s}$ and $r_{p}$ are unknown. Equation (8) satisfies Eq. (3).

The primitive field phasors in $\mathcal{V}_{\text {in }}$ are given as

$$
\left.\begin{array}{l}
\mathbf{E}(\mathbf{r}, \omega)=\left[t_{s} \hat{\mathbf{u}}_{y}+t_{p}\left(-\hat{\mathbf{u}}_{x} \tau+\hat{\mathbf{u}}_{z} \kappa\right) / k\right] \exp (i \tau z) \exp (i \kappa x) \\
\mathbf{B}(\mathbf{r}, \omega)=\frac{k}{\omega}\left[-t_{p} \hat{\mathbf{u}}_{y}+t_{s}\left(-\hat{\mathbf{u}}_{x} \tau+\hat{\mathbf{u}}_{z} \kappa\right) / k\right] \exp (i \tau z) \exp (i \kappa x)
\end{array}\right\}, \quad \mathbf{r} \in \mathcal{V}_{\mathrm{in}},
$$

where $k=\omega \sqrt{\mu \varepsilon}, \tau=+\sqrt{k^{2}-\kappa^{2}}$, and the coefficients $t_{s}$ and $t_{p}$ are unknown. Representing the plane wave refracted into $\mathcal{V}_{\text {in }}$, these expressions satisfy Eq. (5).

The foregoing expressions were substituted into Eqs. (2) $,(4)_{2},(6)_{2,4}$ and (7) $)_{2}$ to determine $r_{s}$, $r_{p}, t_{s}$, and $t_{p}$ in terms of $a_{s}$ and $a_{p}$. Thus,

$$
\begin{gathered}
r_{s}=\frac{\left[\left(\eta_{r}-\delta_{r}\right)\left(1+\eta_{r} \delta_{r}\right)-\left(G \eta_{0}\right)^{2} \eta_{r}^{2} \delta_{r}\right] a_{s}+2 G \eta_{0} \eta_{r}^{2} \delta_{r} a_{p}}{\left(\eta_{r}+\delta_{r}\right)\left(1+\eta_{r} \delta_{r}\right)+\left(G \eta_{0}\right)^{2} \eta_{r}^{2} \delta_{r}}, \\
r_{p}=\frac{\left[\left(\eta_{r}+\delta_{r}\right)\left(1-\eta_{r} \delta_{r}\right)+\left(G \eta_{0}\right)^{2} \eta_{r}^{2} \delta_{r}\right] a_{p}+2 G \eta_{0} \eta_{r}^{2} \delta_{r} a_{s}}{\left(\eta_{r}+\delta_{r}\right)\left(1+\eta_{r} \delta_{r}\right)+\left(G \eta_{0}\right)^{2} \eta_{r}^{2} \delta_{r}}, \\
t_{s}=\frac{2 \eta_{r}\left(1+\eta_{r} \delta_{r}\right) a_{s}+2 G \eta_{0} \eta_{r}^{2} \delta_{r} a_{p}}{\left(\eta_{r}+\delta_{r}\right)\left(1+\eta_{r} \delta_{r}\right)+\left(G \eta_{0}\right)^{2} \eta_{r}^{2} \delta_{r}}, \\
t_{p}=\frac{2 \eta_{r}\left(\eta_{r}+\delta_{r}\right) a_{p}-2 G \eta_{0} \eta_{r}^{2} a_{s}}{\left(\eta_{r}+\delta_{r}\right)\left(1+\eta_{r} \delta_{r}\right)+\left(G \eta_{0}\right)^{2} \eta_{r}^{2} \delta_{r}},
\end{gathered}
$$

where

$$
G=\Psi+\gamma, \quad \eta_{0}=\sqrt{\frac{\mu_{0}}{\varepsilon_{0}}}, \quad \delta_{r}=\frac{\tau / k}{\tau_{0} / k_{0}}, \quad \eta_{r}=\sqrt{\frac{\varepsilon_{0} \mu}{\varepsilon \mu_{0}}} .
$$

We have verified that Eqs. (10) through (13) satisfy Eqs. (6) $)_{1,3}$ and (7) $)_{1}$ Moreover, Eqs. (10) through (13) simplify to the standard results: ${ }^{6,7}$

$$
\left.\begin{array}{ll}
r_{s}=a_{s} \frac{\eta_{r}-\delta_{r}}{\eta_{r}+\delta_{r}}, & r_{p}=a_{p} \frac{1-\eta_{r} \delta_{r}}{1+\eta_{r} \delta_{r}} \\
t_{s}=a_{s} \frac{2 \eta_{r}}{\eta_{r}+\delta_{r}}, & t_{p}=a_{p} \frac{2 r_{r}}{1+\eta_{r} \delta_{r}}
\end{array}\right\}
$$

for $\Psi=\gamma=0$. 


\section{Discussion and Conclusion}

Equations (10) through (13) can be recast in matrix form as

$$
\left[\begin{array}{l}
r_{s} \\
r_{p}
\end{array}\right]=\left[\begin{array}{ll}
r_{s s} & r_{s p} \\
r_{p s} & r_{p p}
\end{array}\right]\left[\begin{array}{l}
a_{s} \\
a_{p}
\end{array}\right], \quad\left[\begin{array}{l}
t_{s} \\
t_{p}
\end{array}\right]=\left[\begin{array}{ll}
t_{s s} & t_{s p} \\
t_{p s} & t_{p p}
\end{array}\right]\left[\begin{array}{l}
a_{s} \\
a_{p}
\end{array}\right] .
$$

The elements of the $2 \times 2$ matrices have either both subscripts identical or two different subscripts. The elements with both subscripts identical indicate copolarized reflection or refraction, with the remaining elements indicating cross polarization. Both cross-polarized reflection and refraction in Eqs. (10) through (13) are due to $G$.

Equations (10) through (13) do not contain $\Psi$ and $\gamma$ separately, but their sum $G$ instead. Thus, measurements of the reflection coefficients $r_{s}$ and $r_{p}$ (or the transmission coefficients $t_{s}$ and $t_{p}$, if at all possible) cannot be used to discriminate between models I $(\gamma=0)$ and II $(\Psi=0)$. Equations $(6)_{4}$ and $(7)_{2}$ together make it clear that measurements of the reflection and transmission coefficients of a slab made of a topological insulator cannot be used to discriminate between the two models; not only that, the solution of every scattering problem will depend on $G$, not on $\Psi$ alone or $\gamma$ alone.

This impasse can be resolved by realizing that surface states exist on topological insulators as protected conducting states, and the characteristic behavior of these materials is due to those surface states. Furthermore, $\Psi$ vanishes from the Maxwell equations (5) applicable to $\mathcal{V}_{\text {in }}$ occupied by the topological insulator; indeed, $\Psi$ would vanish even if the topological insulator were bianisotropic. ${ }^{8}$ For both of these reasons, we must choose model II, which also satisfies the Post constraint $\Psi \equiv 0$. $^{9}$

As the material occupying $\mathcal{V}_{\text {in }}$ is isotropic and achiral, cross-polarized reflection in this problem has been taken to arise from the Lorentz nonreciprocity inherent in Eq. (4). ${ }^{10}$ But now we see that surface states described by Eq. (7) by themselves are capable of yielding cross-polarized reflection, which is, therefore, not an indication of Lorentz nonreciprocity.

\section{Acknowledgments}

A.L. is grateful to the Charles Godfrey Binder Endowment at Penn State for ongoing support of his research. T.G.M. acknowledges the support of EPSRC grant EP/M018075/1.

\section{References}

1. M. Z. Hasan and C. L. Kane, "Topological insulators," Rev. Modern Phys. 82(4), 30453067 (2010).

2. M.-C. Chang and M.-F. Yang, "Optical signature of topological insulators," Phys. Rev. B 80(11), 113304 (2009).

3. F. Liu et al., "Goos-Hänchen and Imbert-Fedorov shifts at the interface of ordinary dielectric and topological insulator," J. Opt. Soc. Am. B 30(5), 735-741 (2013).

4. F. Liu, J. Xu, and Y. Yang, "Polarization conversion of reflected electromagnetic wave from topological insulator,” J. Opt. Soc. Am. B 31(4), 735-741 (2014).

5. J. G. Van Bladel, Electromagnetic Fields, 2nd ed., IEEE Press, Piscataway, New Jersey (2007).

6. A. Lakhtakia, "On pathological conditions and Fresnel coefficients," Int. J. Infrared Millimeter Waves 11(12), 1407-1413 (1990).

7. M. F. Iskander, Electromagnetic Fields and Waves, 2nd ed., Waveland Press, Long Grove, Illinois (2013).

8. A. Lakhtakia and T. G. Mackay, "Axions, surface states, and the Post constraint in electromagnetics," Proc. SPIE 9558, 95580C (2015).

9. E. J. Post, Formal Structure of Electromagnetics, North Holland, Amsterdam, The Netherlands (1962).

10. C. M. Krowne, "Electromagnetic theorems for complex anisotropic media," IEEE Trans. Antennas Propag. 32(11), 1224-1230 (1984). 
Akhlesh Lakhtakia received his degrees from the Banaras Hindu University and the University of Utah. He is the Charles Godfrey Binder Professor of engineering science and mechanics at the Pennsylvania State University. His current research interests include nanotechnology, bioreplication, forensic science, solar-energy harvesting, surface multiplasmonics, metamaterials, mimumes, and sculptured thin films. He is a fellow of OSA, SPIE, IoP, AAAS, APS, and IEEE. He received the 2010 SPIE Technical Achievement Award.

Tom G. Mackay is a reader in applied mathematics at the University of Edinburgh and an adjunct professor in the Department of Engineering Science and Mechanics at the Pennsylvania State University. He is a graduate of the Universities of Edinburgh, Glasgow, and Strathclyde, and a fellow of the Institute of Physics (UK) and SPIE. His research interests include the electromagnetic theory of novel and complex materials, including homogenized composite materials. 